\title{
Editorial
}

\section{Green Communication and Networking}

\author{
Yongpeng Wu $\mathbb{D},{ }^{1}$ Fuhui Zhou, ${ }^{2}$ Zan Li $\left(\mathbb{D},{ }^{3}\right.$ Shunqing Zhang, \\ Zheng Chu, ${ }^{5}$ and Wolfgang H. Gerstacker ${ }^{6}$
}

${ }^{1}$ Shanghai Key Laboratory of Navigation and Location Based Services, Shanghai Jiao Tong University, Minhang, 200240, China

${ }^{2}$ The School of Information Engineering, Nanchang University, 330031, China

${ }^{3}$ The Integrated Service Networks Lab of Xidian University, Xian, 710071, China

${ }^{4}$ The Shanghai Institute for Advanced Communication and Data Science, Shanghai University, Shanghai, 200444, China

${ }^{5}$ The 5 G Innovation Centre, Institute for Communication Systems, University of Surrey, Guildford GU2 7XH, UK

${ }^{6}$ The Institute for Digital Communications of Friedrich-Alexander University, Erlangen, 91054, Germany

Correspondence should be addressed to Yongpeng Wu; yongpeng.wu2016@gmail.com

Received 13 August 2018; Accepted 13 August 2018; Published 2 September 2018

Copyright ( 2018 Yongpeng Wu et al. This is an open access article distributed under the Creative Commons Attribution License, which permits unrestricted use, distribution, and reproduction in any medium, provided the original work is properly cited.

Energy crisis and rising concerns on Greenhouse Gas (GHG) emissions have always been crucial issues faced by the development of wireless communication techniques. As a more energy-efficient network architecture, green communications and networks (GCN) have recently attracted significant attention from academia and industry. In particular, the newly designed GCN not only can alleviate the greenhouse effect and decrease the operational expenditure but also can attain sustainable development due to the descending independence on fossil fuel and the exploitation of renewable energy resources. In order to enable the technical and economical GCNs, several emerging techniques have been proposed including energy-efficient and energy harvesting techniques. Although these emerging techniques have drawn considerable attention and have been studied recently, there are still many open theoretical and practical problems to be addressed. Specifically, most of the existing works have focused on optimizing a single objective of GCNs, such as energy efficiency. Since there are multiple conflicting objectives in GCNs, e.g., spectral efficiency and energy efficiency, multiobjective strategies are required to be in order to achieve a good tradeoff among the conflicting objectives. Moreover, since considered nonorthogonal multiple access techniques have advantages in energy efficiency and massive connectivity, how to apply nonorthogonal multiple access techniques into GCNs needs to be further investigated. Furthermore, the conventional linear energy harvesting model is ideal in practice. How to design GCNs under practical nonlinear energy harvesting models is required to be focused.

This special issue aims to provide a comprehensive overview of the state of the art in theory and practice for realizing GCNs, which will bring together researchers from academia, industry, and governmental agencies to promote the research and development needed to address the major challenges that pertain to this cutting-edge research topic.

(i) Mutliobjective resource allocation strategies for GCNs

(ii) Mutliobjective optimization theory for GCNs

(iii) Mutliobjective energy-efficient techniques for GCNs

(iv) Energy-efficient cooperative techniques for GCNs

(v) Energy-efficient nonorthogonal multiple access techniques for GCNs

(vi) Practical energy harvesting models for GCNs

(vii) Resource optimization for GCNs under practical nonlinear energy harvesting models

(viii) Physical layer security techniques for GCNs

(ix) Multiantenna techniques for GCNs

Twenty-five papers were submitted for this special issue. Our distinguished reviewers from respective research fields narrowed the field to fourteen papers which were finally 
accepted. The following is a short summary of the findings of each of these papers.

T. Wang et al. studied an energy efficiency maximization for an orthogonal frequency division multiple access downlink network aided by a relay station with subcarrier pairing. A resource allocation scheme was proposed to maximize the energy efficiency. A resource allocation algorithm was proposed to solve the formulated problem. Simulation results have shown the impact of minimum required rate of the network on the energy efficiency of the network.

Z. Y. Liu et al. have proposed an energy-efficient incentives resource allocation scheme for a cooperative communication system. The energy efficiency was maximized by the proposed scheme. Moreover, an improved Intelligent Water Drops algorithm combined with Genetic Algorithm. Simulation results demonstrate that the proposed resource allocation scheme can effectively improve the energy efficiency.

Z. Yang et al. have studied a green cognitive radio network. The energy efficiency maximized problems were studied in delay-insensitive green cognitive radio and delaysensitive green cognitive radio. Two algorithms based on the proposed resource allocation strategies were proposed to solve the formulated problems. Simulation results have shown that the maximum energy efficiency of the secondary user achieved under the average transmit power constraint is higher than that achieved under the peak transmit power constraint.

$\mathrm{X}$. Peng et al. have proposed a joint user association and power allocation to maximize the energy efficiency of dense small cell networks. A dynamic coordinated multipoint joint transmission algorithm was proposed to improve energy efficiency. The proposed solution has a much lower computational complexity. Simulation results have shown that the proposed solution has a better performance.

W. Tan et al. have studied the hybrid architecture of multiuser massive MIMO systems. The digital domain utilized the zero-forcing precoding scheme and the analog domain used discrete Fourier transform processing to significantly reduce hardware cost and energy consumption. The analytical expressions on the total achievable spectral efficiency and energy efficiency were derived. The total achievable energy efficiency of hybrid architectures with discrete Fourier transform processing outperforms other massive MIMO architectures.

T. Wang et al. studied an energy-constrained twoway multiplicative amplify-and-forward relay network with simultaneous wireless information and power transfer. A practical nonlinear energy harvesting model was considered. The outage throughput was maximized by the proposed resource allocation scheme. Simulation results have shown the superiority of proposed the dynamic power splitting strategy in terms of the outage throughput.

$\mathrm{X}$. Bai et al. designed a power-splitting scheme in an amplify-and-forward energy-constrained relay system with simultaneous wireless information and power transfer in the presence of a direct link between the source and the destination. A practical nonlinear energy harvesting (EH) model was considered. The authors have proposed a resource allocation scheme for maximizing the system capacity. Simulation results have demonstrated that a higher system capacity can be achieved when the PS scheme is optimized based on nonlinear EH models instead of the linear EH model

$\mathrm{W}$. Ge et al. have studied multiple-input single-output secured cognitive radio networks relying on simultaneous wireless information and power transfer. An AN-aided transmit beamforming was optimized to improve the security of the studied networks. The transmit power of the information signal was minimized subject to the secrecy rate constraint, the harvested energy constraint, and the total transmit power. Simulation results have validated the performance of the proposed algorithms.

F. Engmann et al. have presented a survey paper for the current techniques for prolonging the lifetime of wireless sensor networks. This paper presented the state of the art in the energy management schemes, such as energy harvesting, energy transfer, and energy conservation methods. The remaining challenges and the open issues for future research work were discussed.

B. Kong et al. studied radar and communication systems with orthogonal frequency division multiplexing. This system are promising in the next generation wireless communication due to its high energy efficiency and spectral efficiency. The authors have proposed a joint Range-Doppler-Angle Estimation scheme. A parameterized rectification method was proposed to incorporate the inherent structures of the factor matrices. The numerical experiments have demonstrated superior performance of the proposed algorithm compared with the existing methods.

$\mathrm{W}$. $\mathrm{Hu}$ et al. studied a cooperative relaying wireless communication networks. The physical layer security of a multisource multirelay cooperative relaying communication network was investigated by considering the influence of cochannel interference from a security-reliability tradeoff perspective. Theoretical and simulation results have shown that a better security-reliability tradeoff performance can be achieved by increasing the number of sources, relays, and cochannel interferers.

D. Qin et al. studied an exact average symbol error probability for amplify and forward relaying in independent Nakagami-m fading environments. The closed-form symbol error probability was expressed in the form of Lauricella multivariate hypergeometric function. Four modulation modes were studied. Simulation results have verified the theoretical analysis.

Z. Zheng et al. have presented performance and power consumption analysis of IEEE802.11ah for smart grid. The authors have simulated the new added highly robust $1 \mathrm{MHz}$ bandwidth and Modulation Coding Scheme 10 in the 802.11ah physical layer and analyzed the coverage range and energy saving performance of 802.11ah based on the simulation results. The analysis has shown that the 802.11ah at 1 MHZ MCS 10 mode can obtain 2.5-3 dB gain.

$\mathrm{X}$. Zhang et al. proposed a context-aware location differential perturbation scheme for privacy-aware users in mobile environment. It can enhance the user's location privacy without requiring a trusted third party. This improves the operational efficiency of the system. The proposed scheme 
can provide strong privacy guarantees with a bounded accuracy loss while improving retrieval accuracy.

\section{Conflicts of Interest}

The authors declare that there are no conflicts of interest regarding the publication of this article.

Yongpeng Wu
Fuhui Zhou
Zan Li
Shunqing Zhang
Zheng Chu
Wolfgang H. Gerstacker




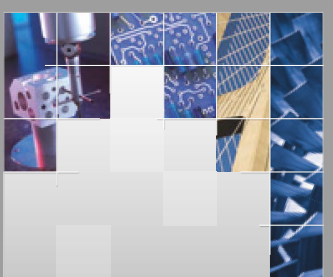

\section{Enfincering}
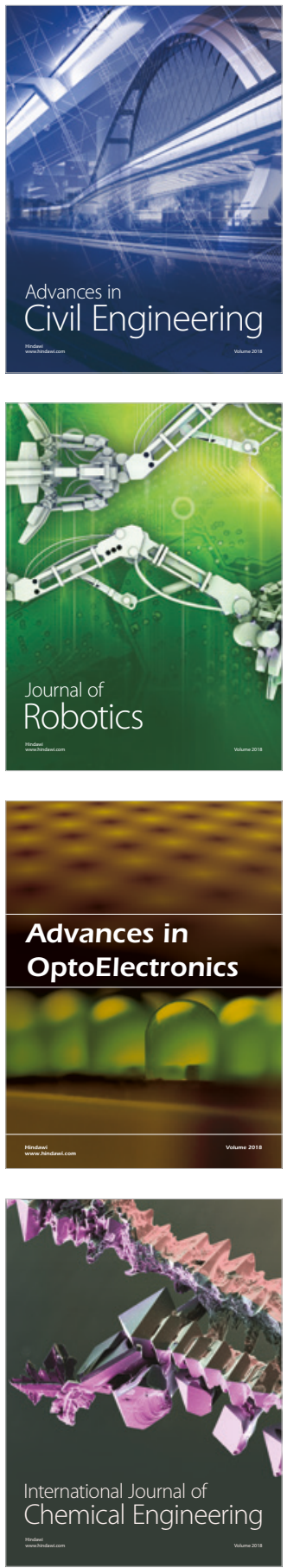

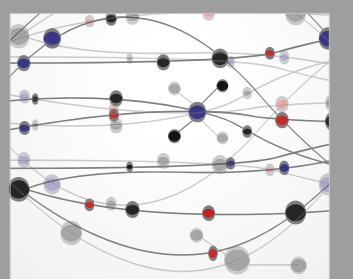

\section{Rotating \\ Machinery}

The Scientific World Journal

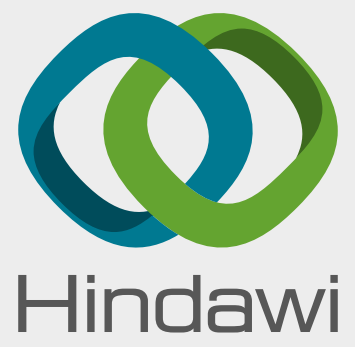

Submit your manuscripts at

www.hindawi.com
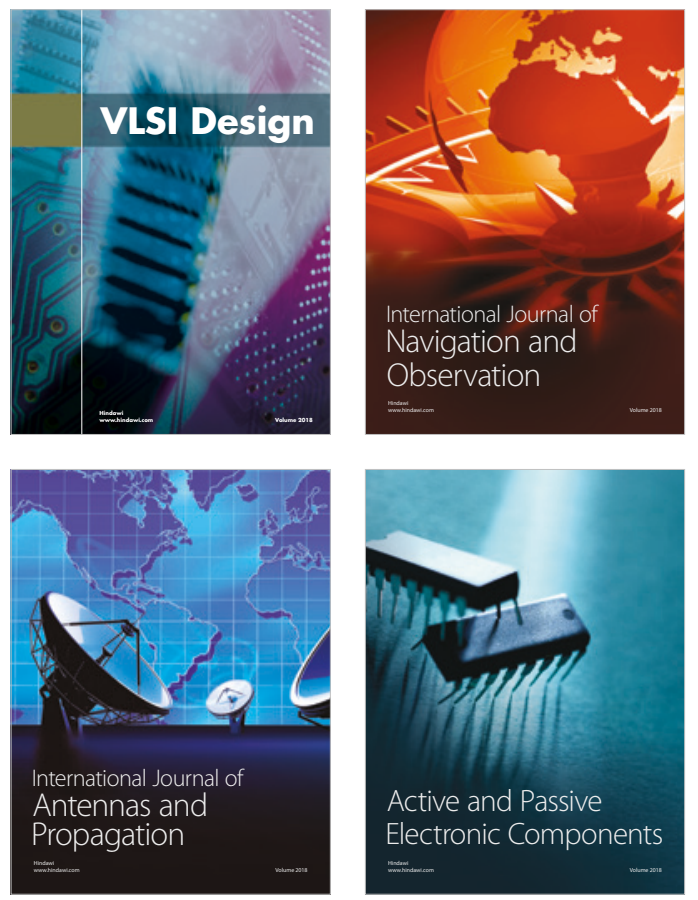
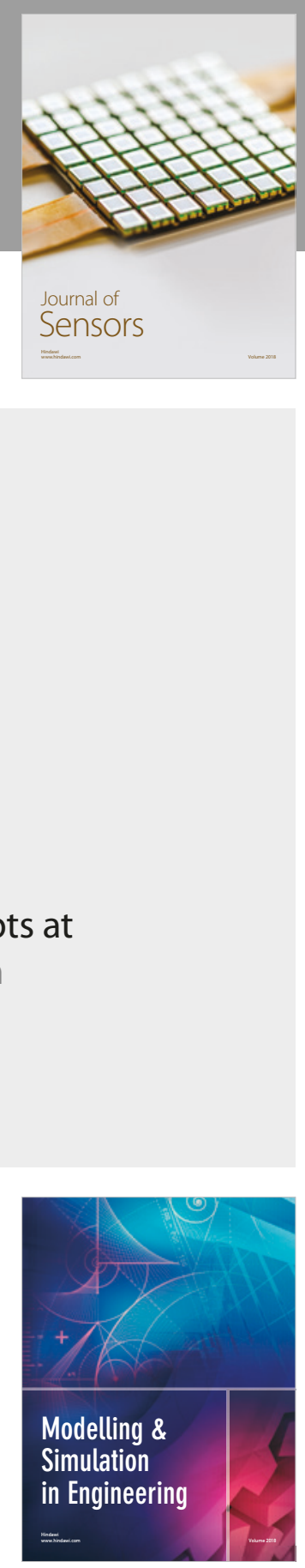

\section{Advances \\ Multimedia}
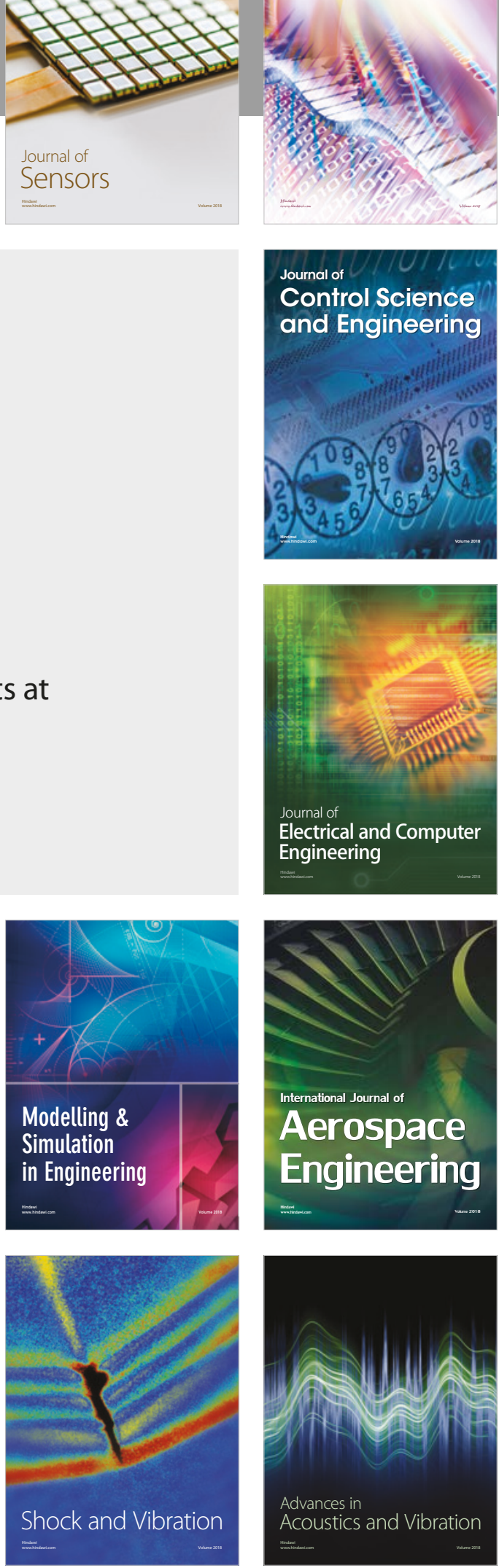